\title{
ABUNDANCIA Y BIOMASA DE CHAETOGNATHA (APHRAGMOPHORA) EN LA ZONA PELÁGICA DEL PACÍFICO COLOMBIANO
}

\section{CHAETOGNATHA (APHRAGMOPHORA) ABUNDANCE AND BIOMASS IN THE EPIPELAGIC ZONE OF THE COLOMBIAN PACIFIC}

\author{
Raúl Hernando López Peralta ${ }^{1}$
}

${ }^{1}$ Biólogo Marino, Dr. rer. nat., Profesor Asociado, Programa de Biología Aplicada, Facultad de Ciencias Básicas y Aplicadas. Universidad Militar Nueva Granada, Campus Cajicá, km 2 vía Cajicá-Zipaquirá, e-mail: raul.lopez@unimilitar.edu.co

\author{
Rev. U.D.C.A Act. \& Div. Cient. 21(1): 187-196, Enero-Junio, 2018 \\ https://doi.org/10.31910/rudca.v21.n1.2018.677
}

\section{RESUMEN}

Dada la importancia de los quetognatos por su alto impacto depredador y su rol en la transferencia energética desde el fitoplancton hasta niveles tróficos superiores, por primera vez, se evaluó en el Océano Pacífico Colombiano (OPC) su biomasa seca, además de su abundancia, en relación con la estructura termohalina y la influencia de la luz, durante septiembre de 2007. Se analizaron 23 muestras obtenidas con una red bongo, mediante arrastres oblicuos en el estrato epipelágico de sendas estaciones, distribuidas en toda el área. Las condiciones abióticas fueron características de una época normal, con posible beneficio para la comunidad de los quetognatos, en respuesta a la disponibilidad de alimento. Mientras que los adultos dominaron en las sub-áreas intermedia $\left(\bar{x}=240\right.$ ind. $\left./ 100 \mathrm{~m}^{3}, 34,7 \mathrm{~g} / 100 \mathrm{~m}^{3}\right)$ y oceánica $\left(\bar{x}=290\right.$ ind. $\left./ 100 \mathrm{~m}^{3} 39,2 \mathrm{~g} / 100 \mathrm{~m}^{3}\right)$, las aguas costeras, generalmente más productivas, pudieron promover el desove, el desarrollo y el mayor biomasa-abundancia de los juveniles (910ind. $/ 100 \mathrm{~m}^{3}, 26,2 \mathrm{~g} / 100 \mathrm{~m}^{3}$ ), sugiriendo su mejor adaptación a aguas menos salinas, así como un impacto trófico más marcado y un papel más relevante, que los adultos en la estructura de la comunidad zooplanctónica. Es muy probable que la dinámica hidrológica haya influido en la distribución heterogénea de los organismos en la zona epipelágica, con biomasa-abundancia más alta en algunas estaciones costeras y oceánicas, debido a la mayor oferta trófica resultante de la conjunción de procesos de surgencia, migración vertical nocturna e influencia lunar, en especial, durante luna nueva y cuarto creciente. Se da una lista de verificación preliminar de los quetognatos.

Palabras clave: Biomasa, abundancia, surgencia, migración, quetognatos.

\section{SUMMARY}

Due to the importance of chaetognaths, their high predatory impact and their role in energy transfer from phytoplankton to higher trophic levels, their abundance and dry biomass were evaluated for the first time in relation to thermohaline structure and light influence in the Colombian Pacific Ocean (CPO) in September 2007. Twenty-three samples were obtained with a bongo net $(294 \mu \mathrm{m}$ mesh) by oblique trawls in the epipelagic stratum in equal number of stations distributed throughout the CPO. Abiotic conditions were characteristic of a normal period, with possible benefit to the chaetognath community as a result of food availability. While adults dominated in the intermediate $\left(\bar{x}=240 \mathrm{ind} / 100 \mathrm{~m}^{3}\right.$, $\left.34.7 \mathrm{~g} / 100 \mathrm{~m}^{3}\right)$ and oceanic ( $\bar{x}=290 \mathrm{ind} / 100 \mathrm{~m}^{3} 39.2 \mathrm{~g} / 100$ $\mathrm{m}^{3}$ ) subareas, coastal waters, generally more productive, promoted the spawning, development and higher abundance and biomass of juveniles (910 ind/100 $\mathrm{m}^{3}, 26.2 \mathrm{~g} / 100 \mathrm{~m}^{3}$ ), which indicates better adaptation to less saline waters, as well as a more pronounced trophic impact and relevant role to the structure of the zooplankton community when compared to the adult community. It is likely that hydrological dynamics have influenced heterogeneous organism distribution on the epipelagic zone, with a higher biomass-abundance in some coastal and oceanic stations, due to greater trophic supply resulting from upwelling processes, nocturnal vertical migration, and lunar influence, especially during the new moon and first quarter. A preliminary checklist of the chaetognaths is given.

Key words: Biomass, abundance, upwelling, migration, chaetognaths. 


\section{INTRODUCCIÓN}

Las comunidades planctónicas son de importancia, por ser la base de las redes tróficas acuáticas y las pesquerías (Kruse et al. 2010; Cota-Meza et al. 2015). Los quetognatos son hermafroditas semi-gelatinosos (Villenas et al. 2009) marinos y el segundo grupo holoplanctónico más profuso, después de los copépodos, su principal presa, dentro de su amplia dieta, que incluye huevos y larvas de peces, por lo que son útiles en los estudios pesqueros (Kruse et al. 2010; Cota-Meza et al. 2015). En general, tienen un papel significativo en la captura del carbono y el transporte de energía (Villenas et al. 2009), mediado por sus migraciones verticales tróficas y ontogénicas circadianas. Varias especies son indicadoras hidrológicas (Ulloa et al. 2000; Hernández et al. 2001; Schramm \& College, 2009; Vereshchaka et al. 2017).

La biomasa y la abundancia del zooplancton son cardinales para interpretar su distribución (Ré et al. 2005; Suthers \& Rissik, 2009). La biomasa ayuda a comprender la dinámica del flujo energético en la trama trófica, estimar los flujos de carbono y de nitrógeno (Villenas et al. 2009) y alimentar modelos matemáticos en la evaluación de la dinámica ecológica del plancton (Rehim et al. 2016). El peso seco y la materia orgánica constituyen la estimación más precisa de la biomasa (Ré et al. 2005; Suthers \& Rissik, 2009), pese, a lo cual, se acostumbra optar por la biomasa húmeda o volumétrica, cuya información sobre los quetognatos es aún exigua en el Pacífico Americano, e. g., Estados Unidos (Lavaniegos \& Ohman, 2007), Costa Rica (Hossfeld, 1996; von Wangelin \& Wolff, 1996), Chile (Villenas et al. 2009) y México (PelayoMartínez et al. 2017).

En el Océano Pacífico Colombiano (OPC) no se había evaluado la biomasa de estos zoopláncteres, pero sí su abundancia y distribución, de manera muy esporádica, desde hace cuatro décadas (López, 1997; Gómez \& López, 2002; López et al. 2007; García et al. 2008; Carvajal et al. 2009).

El objetivo de este estudio fue describir, por primera vez, la distribución espacial de la biomasa de los quetognatos en el OPC, comparada con su abundancia y proporcionar información de referencia para proyectar estudios más detallados de ecología trófica y flujos de carbono. Se planteó la hipótesis de que las biomasas de los quetognatos difieren de su distribución espacial numérica, incluso, entre juveniles y adultos, en particular, en respuesta a la temperatura y la salinidad.

\section{MATERIALES Y MÉTODOS}

Área de estudio: El Océano Pacífico Colombiano (OPC), se enmarca en las coordenadas $01^{\circ} 30^{\prime}-6^{\circ} 30^{\prime} \mathrm{N}$ y $77^{\circ} 45^{\prime}-84^{\circ} 00^{\prime}$ O y limita al sur con Ecuador, al norte con Panamá y al orien- te con la costa colombiana. El clima es ecuatorial cálido, con una humedad relativa del $90 \%$ de saturación y de temperatura $>26^{\circ} \mathrm{C}$. El desplazamiento de la Zona de Convergencia Intertropical (ZCIT) determina las características oceanográficas, como la precipitación, que varía entre 180 y 400 mm, durante enero-marzo y entre 550 y $750 \mathrm{~mm}$, durante abril-diciembre (Giraldo et al. 2014). El principal factor atmosférico modulador de la productividad y la circulación meso-escalar en el OPC es la oscilación estacional de los vientos alisios que, en el primer semestre, favorecen el transporte de agua fría, rica en nutrientes a la zona fótica, incrementando la productividad primaria. Al amainar, en el segundo semestre, limitan el ingreso de aguas cálidas oceánicas, en contraste con el aumento de la precipitación y la reducción de la concentración de nutrientes en la superficie (CCCP, 2002). En esa capa, la salinidad varía entre 27,0 y 33,5ups, en sentido costa-océano y la temperatura de manera inversa, de 27,0 a $26,5^{\circ} \mathrm{C}$. Las oscilaciones térmicas presentan dos máximos, en diciembre y en junio, con profundización de la termoclina (hasta ca. 40m), así como dos mínimos, en marzo y en septiembre, cuando la termoclina asciende hacia aguas más superficiales (10-30m) (CCCP, 2002). Es notoria la influencia de las variaciones de la fría Corriente de Humboldt y la cálida Contracorriente Ecuatorial, los eventos El Niño y La Niña. Se han identificado zonas de surgencia permanente en aguas costeras y oceánicas, cuya extensión disminuye de agosto a octubre (Málikov \& Villegas, 2010; Villegas et al. 2016).

Métodos de campo: El crucero oceanográfico, se efectuó durante la época de lluvias, del 4 al 28 de septiembre de 2007. El zooplancton, se capturó en 23 estaciones, de las 113 que componen la red del Estudio Regional del Fenómeno El Niño (ERFEN-Colombia), cuya denominación numérica se mantuvo, para facilitar la comparación con otros trabajos. Los muestreos fueron diurnos (06:00-18:00 h) y nocturnos (18.01-05.59 h) (Figura 1), dependiendo del arribo del buque a cada estación $(Z=230-3600 \mathrm{~m})$, donde se realizaron arrastres oblicuos en la capa epipelágica hasta una profundidad media de $184 \mathrm{~m}(41,5-262,4 \mathrm{~m})$. Se empleó un equipo bongo $(\varnothing=60 \mathrm{~cm}$, longitud $1,85 \mathrm{~m}$, dos mallas con poro de 294 y $520 \mu \mathrm{m}$, respectivamente), con un flujómetro mecánico Hydro-Bios. Se analizaron las muestras de la primera malla, fijadas en el barco, con formaldehído al $10 \%$, tamponado con borato de sodio (Suthers \& Rissik, 2009). El flujómetro fue calibrado en un tramo marino de $30 \mathrm{~m}$, obteniéndose un factor de calibración $(\mathrm{f})=0,03$, para estimar el volumen de agua filtrada: $\pi \times \mathrm{r}^{2} \mathrm{xd}$, donde $\mathrm{d}=\mathrm{f} \times \mathrm{r}$ (Suthers \& Rissik, 2009). Mediante una sonda Seabird SBE-19, en cada estación, se obtuvieron los datos de temperatura y de conductividad (estimación de la salinidad) en la columna de agua, hasta $1539 \mathrm{~m}$ de profundidad.

Análisis de laboratorio. Abundancia: Dada la gran densidad del zooplancton, con un divisor Folsom, se obtuvieron 


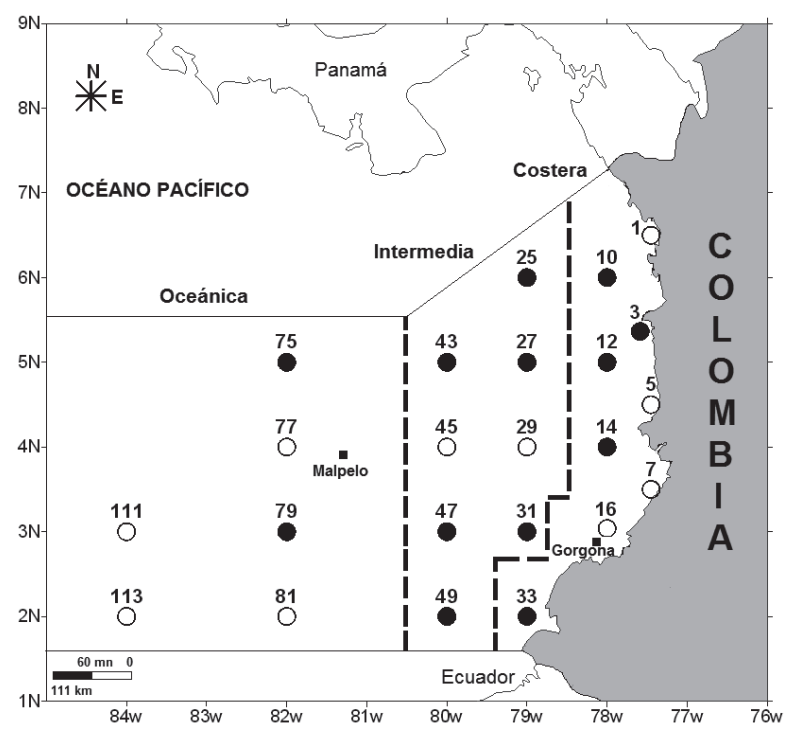

Figura 1. Estaciones para para el muestreo de zooplancton en el océano Pacífico Colombiano, septiembre 2007. ○ estaciones diurnas, • estaciones nocturnas. Numeración correspondiente a la red de estaciones ERFEN-Colombia. Se señalan las sub-áreas costera, intermedia y oceánica.

sub-muestras $(1 / 2,1 / 4,1 / 8$, etc.) de cada estación, previa calibración del aparato (Suthers \& Rissik, 2009). Con base en Sell \& Evans (1982), un análisis de Chi-cuadrado $(\mathrm{p}<0,05)(Z a r, 2013)$ sirvió para comprobar la proporción numérica de los organismos en las dos secciones del divisor Folsom $(p=0,27)$. De cada sub-muestra, se extrajeron, al azar, 30 quetognatos juveniles y 30 adultos, como mínimo estadístico recomendado (Zar, 2013) y se preservaron en formaldehído al $4 \%$, tamponado con borato de sodio (Suthers \& Rissik, 2009). La diferenciación de los estadios de desarrollo, se basó en la visibilidad (adultos) o no (juveniles) de las gónadas (Sund, 1959; Alvariño, 1963; Bonilla, 1983; Casanova, 1999).

La abundancia fue expresada por $100 \mathrm{~m}^{3}$ de agua marina filtrada: $\mathrm{N}=100 \mathrm{~m}^{3} \times(\mathrm{n} \times \mathrm{NF}) / \mathrm{p} \mathrm{x}^{2} \times \mathrm{d}$, donde: $\mathrm{N}=$ Número de individuos/arrastre; $n=$ número de individuos contados; $\mathrm{NF}=$ Número de divisiones Folsom; $r=$ radio de la red; $\mathrm{r}^{2}$ $\mathrm{x} d=$ Volumen de agua filtrada en cada arrastre; $d=1 . \cos$ $(\tan -1 \cdot \tan \mathrm{P})=$ profundidad máxima del lance; coseno y tangente referidas al ángulo del cable, l= longitud máxima $(\mathrm{m})$ del cable filado y $\tan \mathrm{P}=$ tangente promedio del ángulo del cable, medido cada 30 segundos (Suthers \& Rissik, 2009).

Biomasa seca: Para establecer la biomasa, se extrajeron las sales de los especímenes, lavándolos 10 a $15 \mathrm{~s}$, con agua destilada sobre un tamiz (poro $=0,2 \mathrm{~mm}$ ); el intersticial, se eliminó, disponiéndolos sobre papel secante $1 \mathrm{~min}$. (Lovegrove, 1966). Se asignaron sendas formas geométricas, equivalentes a las tres secciones corporales de los quetognatos: cabeza $=$ elipsoide $(4 / 3) \pi \mathrm{x} r 1 \times \mathrm{r} 2 \times \mathrm{r} 3$, donde $\mathrm{r}_{\mathrm{i}}$ es el ancho en las tres dimensiones; cuerpo $=$ cilindro $\pi{ }^{*} \mathrm{r}^{2} * \mathrm{l}$, donde, $r^{2}$ es el ancho y l, la longitud; cola $=$ cono $(1 / 3) \pi$ $\mathrm{x} \mathrm{r}^{2} \mathrm{x} \mathrm{h}$, donde $\mathrm{r}^{2}$ es el ancho en el septum y l, la longitud. Las mediciones, se realizaron con un micrómetro ocular dispuesto en un microscopio. A un horno, se llevaron los organismos hasta peso seco constante $\left(60^{\circ} \mathrm{C}, 24 \mathrm{~h}\right)$; la diferencia entre éste y el peso húmedo equivale a la biomasa seca $(\mathrm{g})$, determinada de inmediato para evitar la rehidratación (Lovegrove, 1966) y asegurar la eliminación de agua, sin afectar el contenido orgánico (Ré et al. 2005). En todos los casos, se usó una balanza analítica de precisión 0,0001g. La biomasa, se estandarizó a $100 \mathrm{~m}^{3}$ al igual que la abundancia (Suthers \& Rissik, 2009).

Lista de verificación: Se incluyó una lista de los quetognatos identificados (Sund, 1959; Alvariño, 1963; Bonilla, 1983; Casanova, 1999), con el fin de contextualizar, preliminarmente, la composición taxonómica, durante septiembre de 2007.

Análisis estadístico: Puesto que las capturas de zooplancton fueron oblicuas, se usaron sus valores promedio, así como los de temperatura $\left({ }^{\circ} \mathrm{C}\right)$ y salinidad (unidades prácticas de salinidad $=$ ups), en la columna de agua definida por la profundidad del muestreo en cada estación. Se aplicaron análisis de componentes principales y agrupamiento (dendrograma modo-Q), para reducir los datos, facilitar su interpretación e identificar grupos con requerimientos ecológicos 
análogos (Zar, 2013). Junto con las biomasas-abundancias en las sub-áreas costera, intermedia y oceánica, se consideraron la temperatura, la salinidad, la intensidad de la luz (variación nictemeral y fases lunares) y los estados juvenil y adulto, por ser los principales parámetros que influyen en la distribución de estos zoopláncteres (Kruse et al. 2010; Cota-Meza et al. 2015; Vereshchaka et al. 2017). Para reducir el rango de los datos bióticos, antes del análisis, se transformaron con $\log (x+1)$; además, se emplearon las pruebas Mann-Whitney y Kruskal-Wallis ( $<<0,05$; nivel de confianza 95,0\%) (Zar, 2013), la primera, para evaluar la variabilidad en los factores bióticos y abióticos y la segunda, para comparar la estructura termohalina entre las sub-áreas. En estos análisis, se empleó el programa Statgraphics Centurion V 16.1®

\section{RESULTADOS Y DISCUSIÓN}

Estructura termohalina de las masas de agua: Los promedios en el estrato muestreado $(41,5-262,4 \mathrm{~m})$ mostraron intervalos moderados de temperatura $\left(20,4-24,7 \pm 0,4^{\circ} \mathrm{C}\right)$ y de salinidad (31,5-33,6 $\pm 0,2$ ups) en aguas costeras y más estrechos en aguas intermedias $\left(20,9-22,9 \pm 0,3^{\circ} \mathrm{C}\right.$ y $32,9-33,8 \pm 0,1$ ups) y oceánicas $\left(20,3-21,9 \pm 0,3^{\circ} \mathrm{C}\right.$ y $33,8-$ $34,3 \pm 0,1$ ups) y se observó un gradiente costa-océano, de menor a mayor salinidad, inverso al gradiente térmico. Esta estructura es típica de una época normal, $i$. e., sin eventos anómalos: El Niño o La Niña (CCCP, 2002; Málikov \& Villegas, 2010; Villegas et al. 2016), cuyas condiciones favorecen la comunidad de los quetognatos, en respuesta a la buena oferta de alimento zooplanctónico (López, 1997; Gómez \& López, 2002; López et al. 2007; García et al. 2008; Carvajal et al. 2009; López, 2012; Jaimes \& López, 2014). No se obtuvieron diferencias significativas entre las tres sub-áreas (Kruskal-Wallis H: 2,70, p>0,05), atribuido a la poca amplitud de los rangos termohalinos.

Distribución de las biomasas y abundancias: Por ser el único registro a la fecha para el OPC, se considera importante anotar que los juveniles y los adultos tuvieron elevados contenidos de agua ( $\bar{x}=93$ y $94 \%, i$. e., 0,7 y $0,6 \%$ de biomasa seca, respectivamente).

Los quetognatos, se hallaron repartidos en todas las estaciones de muestreo, hecho consecuente con su amplia distribución cosmopolita en la columna de agua y todo tipo de aguas marinas (e. g., Lavaniegos \& Ohman, 2007; Hossfeld, 1996; von Wangelin \& Wolff, 1996; Villenas et al. 2009). En general, se capturaron significativamente (Mann-Whitney $p<0,00001)$ muchos más juveniles $\left(22485 \pm 631\right.$ ind./100 ${ }^{3}$, $\mathrm{l}=3,0-14,1 \pm \mathrm{mm})$ que adultos $\left(5479 \pm 149\right.$ ind. $/ 100 \mathrm{~m}^{3}, \mathrm{l}=$ $6,3-22,4 \pm \mathrm{mm})$, con biomasa un poco más alta en los primeros $\left(681,2 \pm 16,8\right.$ us. $\left.635,1 \pm 28,8 \mathrm{~g} / 100100 \mathrm{~m}^{3}\right)$, aunque sin diferencias significativas (Mann-Whitney $p=0,27$ ), entre ambos grupos. La abundancia y la biomasa general de los juveniles/sub-área decrecieron en sentido costa-océano, mientras que los adultos se distribuyeron de manera semejante en todo el OPC, aunque su biomasa fue cerca del $50 \%$ menor en aguas costeras, en relación con el resto del área (Figura 2). Situaciones análogas se han observado en el Golfo Dulce y Golfo de Nicoya del Pacífico costarricense e inmediaciones de la isla San Sebastián (Brasil) (Liang \& Vega-Pérez, 2002). Entre las sub-áreas costera-intermedia-oceánica, no se obtuvieron asociaciones significativas de biomasa de juveniles y de adultos, pero sí de abundancia (Mann-Whitney $p=0,00148,0,00094$ y 0,0083 , respectivamente), consecuentes con los intervalos de estas variables.

Las condiciones propicias en aguas costeras pueden aumentar la probabilidad de que un amplio número de quetognatos juveniles alcance la adultez y sean transportados a áreas alejadas de aquellas por las corrientes, uno de los factores cruciales en la distribución global del plancton (Ulloa et al. 2000; Lavaniegos \& Ohman, 2007), advertido en el OPC antes para los quetognatos y otros zoopláncteres (Gómez \& López, 2002; López et al. 2007; García et al. 2008; Carvajal et al. 2009; López, 2012; Jaimes \& López, 2014). Según Martínez et al. (2007) y Murcia \& Giraldo (2007) es factible que la estructura y la composición del mesozooplancton en esa área dependan, principalmente, de ese factor, como también lo mencionan Ulloa et al. (2000) y Villenas et al. (2009), para las poblaciones de quetognatos en aguas de Chile.

En varios casos, las abundancias y las biomasas más elevadas de juveniles y de adultos confluyeron en zonas de surgencia, tanto oceánicas como costeras del OPC, donde son comunes estos procesos, así como remolinos y grandes aportes de nutrientes, por el gran número de ríos que elevan la productividad biológica (López, 1997; CCCP, 2002; Málikov \& Villegas, 2010; Villegas et al. 2016). Lo expuesto contribuye a comprender la concentración de los quetognatos en áreas de mayor productividad y corresponde a lo expresado por Duró \& Saiz (2000) y Liang \& Vega-Pérez (2002), quienes anotan que, independientemente de la época y de la región, los quetognatos juveniles son dominantes (con excepciones al identificar las especies); además, que su proporción, como la de adultos, se puede relacionar con la presencia de diferentes masas de agua y la estratificación de la columna de agua, dependiendo del periodo del año.

Estos resultados sugieren un impacto trófico más marcado de los individuos inmaduros sobre la estructura de la comunidad zooplanctónica, en contraste con lo observado en el Golfo Dulce y Golfo de Nicoya (Costa Rica), donde se ha observado la predominancia de los adultos, con una fuerte presión depredadora (Hossfeld, 1996; von Wangelin \& Wolff, 1996); no obstante, la preponderancia juvenil en la sub-área costera, en el Mediterráneo español (Duró \& Saiz, 2000) y 

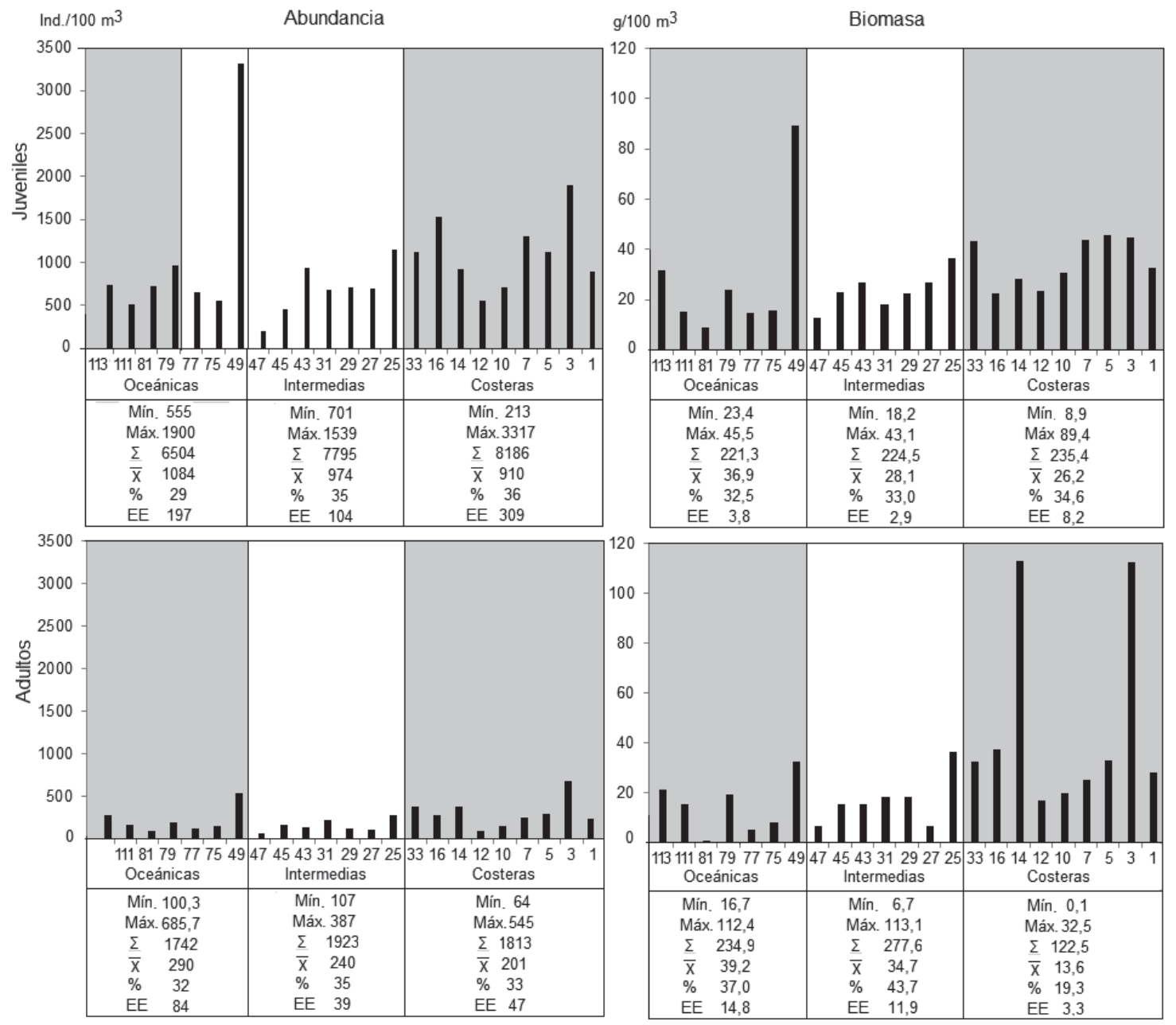

Figura 2. Abundancia (ind. $\left./ 100 \mathrm{~m}^{3}\right)$ y biomasa seca $\left(\mathrm{g} / 100 \mathrm{~m}^{3}\right)$ de quetognatos, en las estaciones de muestreo/sub-área en el OPC, septiembre 2007. El sombreado indica las zonas caracterizadas por procesos de surgencia (Málikov \& Villegas, 2010; Villegas et al. 2016).

proximidades de la isla San Sebastián (Brasil) (Liang \& VegaPérez, 2002), igual a lo encontrado en el OPC, apunta a favorabilidad para el desove y el desarrollo durante la época de muestreo.

En vista de la gran variabilidad espaciotemporal de la comunidad de los quetognatos (Duró \& Saiz, 2000), sus biomasas-abundancias en el OPC pueden advertir oscilaciones temporales más grandes, si se considera el tipo de muestreo, que las capturas no fueron simultáneas y solo se efectuaron durante un mes del periodo lluvioso.

Aunque no se habían obtenido antes valores de biomasa seca de los quetognatos en el OPC, es de notar que las reportadas en el presente trabajo (juveniles y adultos, $8,9-89,4$ y $0,1-113,1 \mathrm{~g} / 100 \mathrm{~m}^{3}$, respectivamente) podrían constituir una proporción relativamente alta de la biomasa seca de zooplancton total (no establecida para septiembre de 2007), si se comparan con los datos de la corriente de Colombia $\left(375-698 \mathrm{~g} / 100 \mathrm{~m}^{3}\right)$, Bahía de Tumaco $\left(0,04-130 \mathrm{~g} / 100 \mathrm{~m}^{3}\right)$ (Velasco, 2011) e inmediaciones de la isla Gorgona $\left(110-280 \mathrm{~g} / 100 \mathrm{~m}^{3}\right.$, en octubre, época de lluvias, us. $190-16870 \mathrm{~g} / 100 \mathrm{~m}^{3}$, en marzo, época menos lluviosa) (Giraldo et al. 2014).

Variación día: noche y ciclo lunar: Pese a la ausencia de diferencias significativas día: noche abundancia (Mann-Whitney $p=0,34$ juveniles, $p=0,34$ adultos) $u s$. biomasa (Mann-Whitney $p=0,67$ juveniles, $p=0,92$ adultos), la proporción nocturna de juveniles fue mucho más alta, mientras que en biomasa apenas sobrepasaron en 3\% la de adultos (Figura 3). Tal vez, durante la noche, los adultos se encontraban en es- 
tratos inferiores a los de captura, dada su mayor capacidad de migración vertical, según anotan Cota-Meza et al. (2015), en su estudio en Bahía Magdalena, Baja California Sur.

En el OPC, López et al. (2007) también reportaron abundancias nocturnas elevadas durante septiembre de 2001 y 2003, a diferencia del mismo mes, en 2002, cuando aumentaron en el día, atribuyéndolo a la influencia de El Niño, moderado imperante durante esa época. Un registro similar de la relación día: noche de la biomasa general de quetognatos $(>30 \mathrm{~mm})\left(46: 41 \mathrm{mg} / 1000 \mathrm{~m}^{3}\right)$ es el de Roger (1994), para el océano Índico. La posible razón de esta relación en el OPC puede ser el desplazamiento nocturno de los organismos a capas superficiales, desde diferentes profundidades, para aprovechar el aumento de alimento, por efecto de las migraciones masivas del zooplancton, evidenciadas en todo el mundo (Ré et al. 2005; Suthers \& Rissik, 2009). En el caso de los quetognatos, éstas se pueden dar en la zona epipelágica e, incluso, desde profundidades superiores a $3.000 \mathrm{~m}$, dependiendo de la especie (Duró \& Saiz, 2000; Ulloa et al. 2000; Cota-Meza et al. 2015).

Adicionalmente, se observaron más altas abundancias y biomasas de los quetognatos juveniles y adultos, en particular de los primeros, durante luna llena, tanto de día como de noche, aunque con notoria representación de los adultos, disminuyendo luego, según las demás fases lunares (Figura 3). El resultado de la prueba Mann-Whitney, significativo para la abundancia $(p=0,002)$, pero no para la biomasa $(p=$ 0,46 ), refleja la mayor amplitud de los rangos numéricos $v s$. los estrechos de biomasa. Igualmente, en aguas costeras de Bonaire (Antillas Holandesas), Schramm \& College (2009) hallaron altas abundancias de quetognatos en los estratos superficiales durante luna llena, así como en luna nueva, el día y la noche.

El comportamiento de los quetognatos con respecto al ciclo lunar en el OPC fue distinto al reportado, como usual, para el zooplancton, de ser menos abundante en la superficie durante luna llena, como respuesta anti-depredadora a otros zoopláncteres carnívoros, peces y aves, con hábitos tróficos nocturnos (Schramm \& College, 2009; Asha et al. 2016). Este patrón, se ha observado, por ejemplo, al norte de Tenerife (islas Canarias), con aumento de los quetognatos y otros zoopláncteres epipelágicos, por la menor presión depredadora del macrozooplancton y el micronecton (Hernández et al. 2001). Hacia la superficie de la misma región, la biomasa seca del mesozooplancton puede ser muy alta en cuarto creciente y disminuir drásticamente tras luna llena, la que se puede elevar en estratos inferiores, con una gran
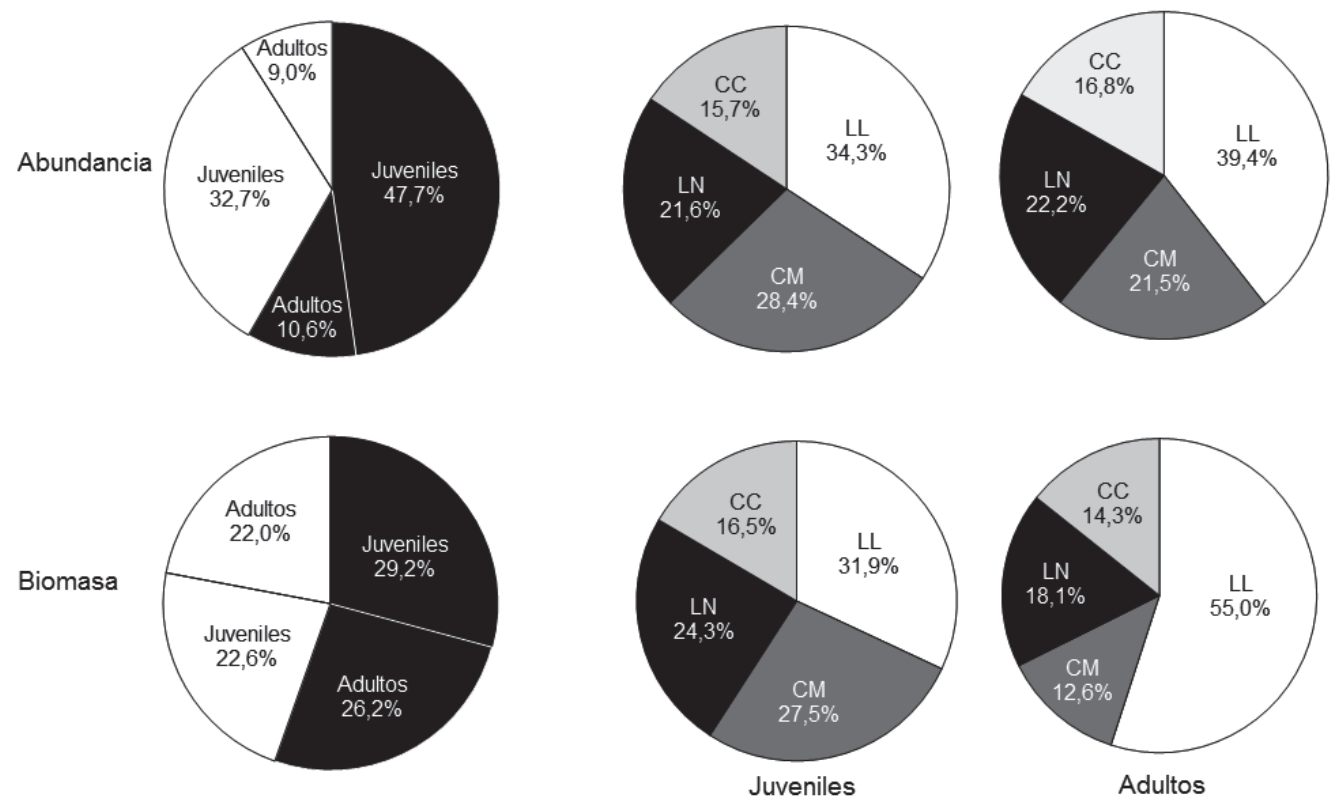

Día $\square$ Noche

LL: luna llena CM: cuarto menguante LN: luna nueva CC: cuarto creciente

Figura 3. Abundancia y biomasa seca de quetognatos (\%), según los periodos día: noche y fases lunares en el OPC, septiembre 2007. 
variabilidad, debida a los sifonóforos y quetognatos grandes

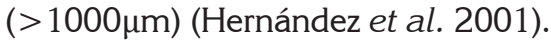

Similar es el caso del macro y mesozooplancton en inmediaciones de las islas Hawaii, cuyas abundancias fluctúan con el nivel de luz, la fase lunar y la profundidad (Benoit-Bird et al. 2009). En el Golfo de Mannar (O. Índico), hacia luna nueva, se ha apreciado un incremento de la abundancia superficial de copépodos, cladóceros y quetognatos, inverso a otros zoopláncteres, e. g., decápodos y larvas de bivalvos, más abundantes durante luna llena (Asha et al. 2016).

En suma, no necesariamente ocurren migraciones más significativas hacia la superficie durante las noches más oscuras de luna nueva, ni es una regla la diferenciación en la distribución vertical entre esta fase lunar y las demás. Tampoco, hay acoplamiento de la luz y el ciclo lunar (Hernández et al. 2001; Benoit-Bird et al. 2009) y, sustancialmente, hay más variabilidad en la migración durante el ciclo, así que este tipo de iluminación puede ser una señal para un ritmo endógeno en el proceso de migración nictemeral, en vez de una causa directa (Benoit-Bird et al. 2009).

Relación entre las variables bióticas y abióticas: Los tres primeros componentes del ACP (Tabla 1) explicaron el 69,6\% de la varianza total $(1=35,8 \%, 2=19,2 \%, 3=14,6 \%)$. Las cargas significativas positivas y negativas corroboraron la influencia termohalina sobre los quetognatos. Del mismo modo, las correlaciones significativas de la abundancia y la biomasa con los periodos día: noche y las fases lunares, en especial, luna nueva y cuarto creciente, confirmaron la posible influencia de la iluminación lunar sobre la comunidad de quetognatos, como en otras regiones del globo (Hernández et al. 2001; Benoit-Bird et al. 2009; Schramm \& College, 2009; Asha et al. 2016). Los grados de asociación obtenidos con el ACP, entre las variables consideradas, son un indicio de la influencia de otros factores en la estructura y en la dinámica de la comunidad de los quetognatos, e. g., reproducción y relaciones tróficas, a lo cual, se han referido Villenas et al. (2009), para el sur de Chile.

El análisis de agrupamiento (Figura 4) mostró el asocio de las estaciones de muestreo en cuatro bloques, según los periodos día: noche y las fases lunares, alguna correspondencia con la ubicación de las estaciones en el OPC y los eventos de surgencia, reportados como permanentes para la época de muestreo (Málikov \& Villegas, 2010; Villegas et al. 2016).

Lista de verificación: Los rangos de longitud entre juveniles y adultos tuvieron un entrecruzamiento, explicado por la normal mezcla de diferentes especies con características morfométricas distintas, según su estadio de desarrollo (Sund, 1959; Alvariño, 1963; Bonilla, 1983; Casanova, 1999). En este sentido, se identificaron adultos de diez especies, correspondientes al $40 \%$ de las 25 reportadas para el OPC (García et al. 2008): Ferosagitta robusta (Doncaster, 1902), Flaccisagitta enflata (Grassi, 1881), Flaccisagitta hexaptera (d'Orbigny, 1836), Krohnitta subtili (Grassi, 1881) Krohnitta pacifica (Aida, 1897), Pterosagitta draco (Krohn, 1853), Sagitta zetesio (Fowler, 1905), Serratosagitta serratoden-

Tabla 1. ACP de las abundancias y biomasas de los quetognatos y las variables abióticas en el OPC, en septiembre de 2007.

\begin{tabular}{|c|l|ccc|}
\hline \multicolumn{2}{|c}{ Variable } & \multicolumn{3}{c|}{ Componente } \\
& \multicolumn{2}{|c|}{$\mathbf{1}$} & $\mathbf{2}$ & $\mathbf{3}$ \\
\hline \multirow{2}{*}{ Abundancia } & adultos & $\mathbf{0 , 4 3}$ & $-0,08$ & 0,15 \\
& juveniles & $\mathbf{0 , 4 0}$ & 0,00 & 0,11 \\
\hline \multirow{2}{*}{ Biomasa } & adultos & $\mathbf{0 , 4 1}$ & $-0,04$ & 0,05 \\
& juveniles & $\mathbf{0 , 4 1}$ & $-0,05$ & 0,06 \\
\hline \multirow{4}{*}{ Fase lunar } & cuarto creciente & $-0,02$ & 0,10 & $\mathbf{0 , 5 2}$ \\
& luna llena & $\mathbf{0 , 3 3}$ & $-0,19$ & $-0,02$ \\
& cuarto menguante & $-0,20$ & $-0,12$ & 0,18 \\
& luna nueva & $-0,11$ & 0,21 & $-\mathbf{0 , 5 9}$ \\
\hline día & $-0,04$ & $\mathbf{- 0 , 6 1}$ & $-0,20$ \\
noche & 0,04 & $\mathbf{0 , 6 1}$ & 0,20 \\
salinidad & $\mathbf{0 , 3 5}$ & 0,04 & $-\mathbf{0 , 3 4}$ \\
temperatura & $-0,19$ & $-\mathbf{0 , 3 8}$ & 0,34 \\
\hline Varianza acumulada & $35,80 \%$ & $19,20 \%$ & $14,60 \%$ \\
\hline
\end{tabular}




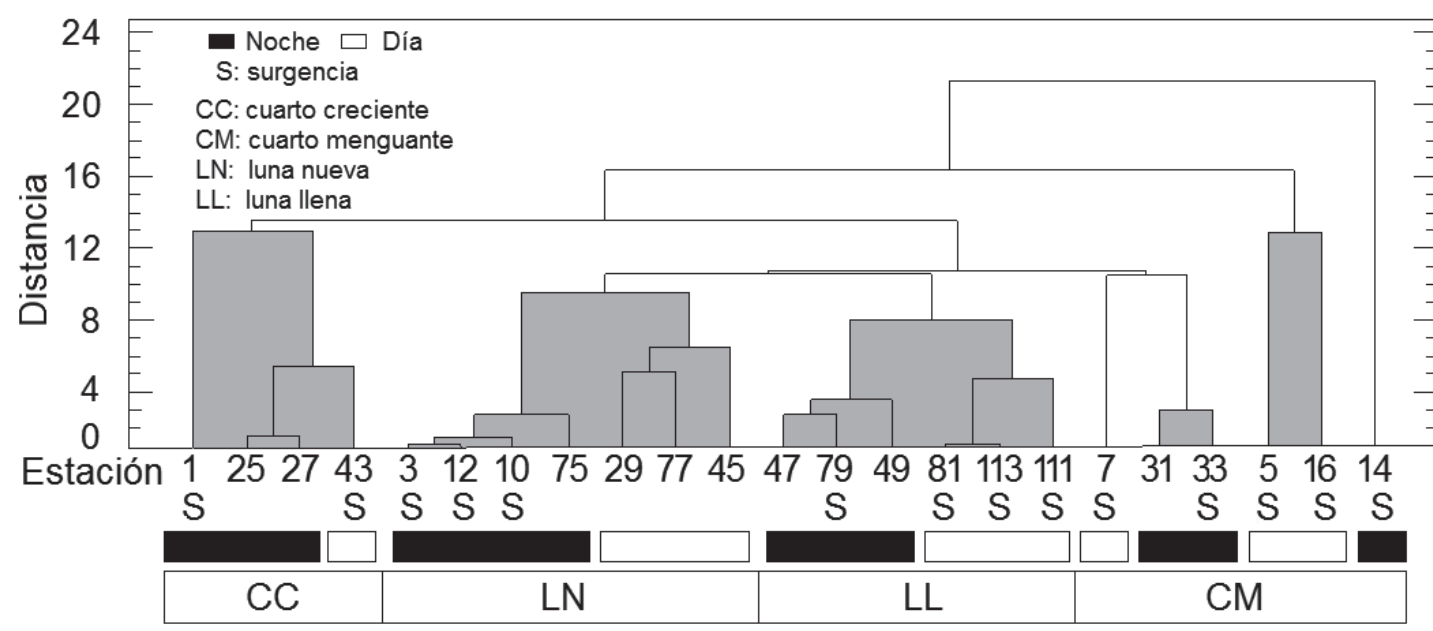

Figura 4. Dendrograma modo-Q mostrando el agrupamiento entre las estaciones de muestreo, con base en las abundancias y biomasas de los quetognatos y las variables abióticas en el OPC, septiembre de 2007. Valor cofenético=0,8.

tat (Krohn, 1853), Serratosagitta pacifica (Tokiota, 1940), Zonosagitta pulchra (Doncaster, 1902). Este porcentaje, se explica porque la identificación, en especial de los especímenes de Sagitta Quoy y Gaimard, 1827, aún se encuentra en proceso de complementación, el cual, incluye la diferenciación de los estadios de desarrollo gonadal por especie, objeto de un trabajo posterior.

En suma, los resultados muestran una comunidad de quetognatos con predominancia de las formas juveniles, en especial, en aguas costeras y en algunos sectores allí y mar adentro, asociados con procesos de alta productividad. Así, es probable su mayor impacto trófico sobre la estructura de la comunidad zooplanctónica, durante un periodo de condiciones abióticas de normalidad, $i$. e., sin eventos El Niño o La Niña. Es de resaltar la influencia de las corrientes, la migración vertical nocturna y la luz lunar en los patrones de distribución de los quetognatos, en lo cual, se debe considerar que en el trópico, las generaciones varían mucho, según la especie, entre ca. 20 días y cuatro semanas, dependiendo de la temperatura (Cota-Meza et al. 2015). Por lo tanto, en investigaciones futuras, se debe profundizar en la identificación de las especies y sus diferentes estadios de desarrollo, en diferentes periodos del año, así como la influencia de otros factores en la estructura y en la dinámica de la comunidad de los quetognatos.

Agradecimientos: El proyecto: "CIAS-2048 Determinación taxonómica y dinámica ecológica de la comunidad de quetognatos (Phylum Chaetognatha) en el océano Pacífico colombiano" fue financiado por la Vicerrectoría de Investigaciones de la UMNG. Agradecimientos a las Biólogas Ángela Baldrich y Gloria Pinto, por su apoyo en las actividades de laboratorio, así como a la Dirección General Marítima (DIMAR) y al Centro de Control de Contaminación del Pacífico (CCCP), por el suministro de las muestras y los datos brutos de temperatura y salinidad. Conflictos de intereses: El manuscrito fue preparado por el autor, quien declara que no existe ningún conflicto de intereses, que ponga en riesgo la validez de los resultados presentados.

\section{BIBLIOGRAFÍA}

1. ALVARIÑO, A. 1963. Quetognatos epiplanctónicos del Mar de Cortés. Rev. Soc. Mex. Hist. Nat. (México). 34:97-203.

2. ASHA, N.P.; SELVARANJITHAM, N.; DIWAKAR, K.; SIVANESH, H.; VIVEKANANDAN, E. 2016. Influence of lunar cycle and hydrological parameters on the depth-wise distribution of zooplankton in the inshore waters off Tuticorin, Gulf of Mannar. J. Mar. Biol. Ass. (India). 58(1):75-81.

3. BENOIT-BIRD, K.J.; WHITLOW, W.L.; AU, W.W.L.; WISDOMA, D.W. 2009. Nocturnal light and lunar cycle effects on diel migration of micronekton. Limnol. Oceanogr. (Estados Unidos). 54(5):1789-1800.

4. BONILLA, D. 1983. Estudio taxonómico de los quetognatos del golfo de Guayaquil. Acta Oceanogr. Pac. (Ecuador). 2(2):512-567.

5. CARVAJAL, L.A.; VERGARA, C.F.; LÓPEZ, R.H. 2009. Chaetognatha, Thaliacea, Euphausiacea and pelagic Polychaeta in the Colombian Pacific Ocean du- 
ring two periods in 1996 (La Niña) and two periods in 1997 (El Niño). Rev. Fac. Cien. Bás. (Colombia). 5(1):173-185.

6. CASANOVA, J. 1999. Chaetognatha. En: Boltovskoy, D. (ed). South Atlantic zooplankton, Backhuys Publishers (Leiden). vol. 2, p.869-1706.

7. CCCP. 2002. Compilación oceanográfica de la Cuenca Pacífica Colombiana. Centro Control Contaminación del Pacífico. Ed. Imágenes de la Naturaleza, Cali (Colombia), 109p.

8. COTA-MEZA, M.S.; FERNÁNDEZ-ÁLAMO, M.A.; FÚNES-RODRÍGUEZ, R. 2015. Abundancia espacio temporal de Flaccisagitta enflata y de la comunidad Chaetognatha en un ciclo circadiano en el Sistema Lagunar de Bahía Magdalena, Baja California Sur, México. Hidrobiológica (México). 25(3):417-426.

9. DURÓ, A.; SAIZ, E. 2000. Distribution and trophic ecology of chaetognaths in the western Mediterranean in relation to an inshore-offshore gradient. J. Plankton Res. (Inglaterra). 22(2):339-361.

10. GARCÍA, X.F.; DE OLIVEIRA L.M.; HERRERA, Y. 2008. Influencia de los eventos climáticos El Niño y La Niña en la comunidad de Chaetognatha de las aguas superficiales del océano Pacífico colombiano. Rev. Bras. Enga. Pesca. (Brasil). 3(1):30-50.

11. GÓMEZ, M.; LÓPEZ, R. 2002. Abundancia y distribución de quetognatos durante los periodos mayo-junio y noviembre de 1996 en el Pacífico Colombiano. Bol. Cien. (Colombia). 7:203-225.

12. GIRALDO, A.; VALENCIA, B.; ACEVEDO, J.D.; RIVERA, M. 2014. Fitoplancton y zooplancton en el área marina protegida de Isla Gorgona, Colombia, y su relación con variables oceanográficas en estaciones lluviosa y seca. Rev. Biol. Trop. (Costa Rica). 62(1):117-132.

13. HERNÁNDEZ, S.; ALMEIDA, C.; YEBRA, L.; ARÍSTEGUI, J.; FERNÁNDEZ DE PUELLES, M.L.; GARCÍA-BRAUIN, J. 2001. Zooplankton abundance in subtropical waters: Is there a lunar cycle? Sci. Mar. (España). 65 (Suppl. 1):59-63.

14. HOSSFELD, B. 1996. Distribution and biomass of arrow worms (Chaetognatha) in Golfo de Nicoya and Golfo Dulce, Costa Rica Rev. Biol. Trop. (Costa Rica). 44. Suppl. 3:157-172.
15. JAIMES, J.C.; LÓPEZ, R.H. 2014. Biomasa y abundancia de Copepoda (Crustacea) en aguas superficiales del océano Pacífico colombiano durante septiembre de 2007. Rev. Bio. Mar. Ocean. (Chile). 49(1):31-41.

16. KRUSE, S.; BREY, T; BATHMANN, U. 2010. Role of midwater chaetognaths in Southern Ocean pelagic energy flow. Mar. Ecol. Prog. Ser. (Alemania). 416:105113.

17. LAVANIEGOS, B.E.; OHMAN, M.D. 2007. Coherence of long-term variations of zooplankton in two sectors of the California Current System. Prog. Oceanogr. (Inglaterra). 75:42-69.

18. LIANG, T.H.; VEGA-PEREZ, L.A. 2002. Distribution, abundance and biomass of chaetognaths off São Sebastião region, Brazil in February 1994. Rev. Bras, Oceanogr. (Brasil). 50:1-12.

19. LÓPEZ, R. 1997. Das Fischplankton der pazifishen Küstenewässer Kolumbiens. Tectum, Marburg. (Alemania). 93:1-522.

20. LÓPEZ, R. 2012. Distribución y abundancia de copépodos pelágicos en el Pacífico colombiano. Rev. Fac. Cien. (Colombia). 8(1):98-123.

21. LÓPEZ, R.H.; LÓPEZ, C.A.; URIBE-PALOMINO, J. 2007. Quetognatos: ċlndicadores de eventos climáticos anómalos en el océano Pacífico colombiano? Bol. Cien. CCCP. (Colombia). 14:109-122.

22. LOVEGROVE, T. 1966. The determination of the dry weight of plankton and the effect of various factors on the values obtained, in some contemporary studies. En: Barnes, H. (ed). Marine Science. Allen \& Unwin, St. Leonards, N.S.W. (Australia). p.429-467.

23. MÁLIKOV, I.; VILLEGAS, N. 2010. Proceso de mezcla vertical en las masas de agua de la Cuenca del Pacífico Colombiano y comportamiento anual de sus características termohalinas. Geo. Col. (Colombia). 35:102-112.

24. MARTÍNEZ, T.I.; GIRALDO, A.; RODRÍGUEZ-RUBIO, E. 2007. Zooplancton en la corriente de Colombia, Pacífico colombiano, durante marzo de 2006). Bol. Cien. CCCP. (Colombia). 14:69-82.

25. MURCIA, M.; GIRALDO, A. 2007. Condiciones oceanográficas y composición del mesozooplancton en la zona oceánica del pacífico colombiano, durante sep- 
tiembre-octubre 2004. Bol. Cien. CCCP (Colombia). 14:83-94.

26. PELAYO-MARTÍNEZ, G.; OLIVOS-ORTIZ, A.; FRANCO-GORDO, C.; QUIJANO-SCHEGGIA, S.; GAVIÑO-RODRÍGUEZ, J.; KONO-MARTÍNEZ, K.; CASTRO-OCHOA, F. 2017. Physical, chemical and zooplankton biomass variability (inshore-offshore) of Mexican Central Pacific during El Niño-La Niña 2010 Lat. Am. J. Aquat. Res. (Chile). 45(1):67-78.

27. RÉ, P.; AZEITEIRO, U.; MORGADO, F. 2005. Ecologia do plâncton marinho e estuarino. Ed. Afrontamento, Porto (Portugal). 140p.

28. REHIM, M.; ZHANG, Z.; MUHAMMADHAJI, A. 2016. Mathematical analysis of a nutrient-plankton system with delay. SpringerPlus (Alemania). 5:1055, 22p. DOI 10.1186/s40064-016-2435-7 Disponible desde internet en researchgate.net. (con acceso 05/01/2018).

29. ROGER, C. 1994. The plankton of the tropical western Indian ocean as a biomass indirectly supporting surface tunas (yellowfin, Thunnus albacares and skipjack, Katsuwonus pelamis). Environ. Biol. Fishes. (Holanda). 39:161-172.

30. SCHRAMM, N.A.; COLLEGE, E. 2009. The effects of the lunar cycle on plankton density, diversity, and diel migration in the coastal waters of Bonaire. Physis (Brasil). 6:27-34.

31. SELL, D.W.; EVANS, M.S. 1982. A statistical analysis of subsampling and an evaluation of the Folsom plankton splitter. Hydrobiologia (Países Bajos). 94(3):223-230.

32. SUND, P.N. 1959. A key to the Chaetognatha of the Tropical Eastern Pacific Ocean. Pac. Sci. (Estados Unidos). 13:269-285.
33. SUTHERS, I.; RISSIK, D. 2009. Plankton: a guide to their ecology and monitoring for water quality. CSIRO Plublishing, Melbourne (Australia). 249p.

34. ULLOA, R.; PALMA, S.; SILVA, N. 2000. Bathymetric distribution of chaetognaths and their associations with water masses of the coast of Valparaíso, Chile. DeepSea Res. (Inglaterra). 47:2009-2027.

35. VELASCO, E. 2011. Estimaciones de la biomasa zooplanctónica en la Bahía de Tumaco durante junio 2009 a junio 2010, Pacífico colombiano. Bol. Cien. $\mathrm{CIOH}$ (Colombia). 29:122-128.

36. VERESHCHAKA, A.; ABYZOVA, G.; LUNINA, A.; MUSAEVA, E. 2017.The deep-sea zooplankton of the North, Central, and South Atlantic: Biomass, abundance, diversity. Deep-Sea Res. II. (Ingl.). 137:89-101.

37. VILLEGAS, N.; MÁLIKOV, I.; DÍAZ, D. 2016. Variabilidad mensual de la velocidad de surgencia y clorofila a en la región del Panama Bight. Mutis (Colombia). 6(2):82-94.

38. VILLENAS, F.; SOTO, D.; PALMA, S. 2009. Cambios interanuales en la biomasa y biodiversidad de zooplancton gelatinoso en aguas interiores de Chiloé, sur de Chile (primaveras 2004 y 2005). Rev. Bio. Mar. Ocean. (Chile). 44(2):309-324.

39. VON WANGELIN, M.; WOLFF, M. 1996. Comparative biomass spectra and species composition of the zooplankton communities in Golfo Dulce and Golfo de Nicoya, Pacific coast of Costa Rica. Rev. Bio. Trop. (Costa Rica). 44(Suppl. 3):135-155.

40. ZAR, J.H. 2013. Biostatistical Analysis. Fifth Edition, Pearson Ed. Ltd. Harlow (Inglaterra). 760p.

Recibido: Febrero 3 de 2018

Aceptado: Marzo 29 de 2018

Cómo citar:

López Peralta, R.H. 2018. Abundancia y biomasa de Chaetognatha (Aphragmophora) en la zona pelágica del pacífico colombiano. Rev. U.D.C.A Act. \& Div. Cient. 21(1): 187-196. 